\title{
Blind, Secured and Robust Watermarking for 3-D Polygon Mesh using Vertex Curvature
}

\author{
Priyanka Singh, K Jyothsna Devi \\ SRM University - A.P \\ Amaravati 522502 \\ A.P, India
}

\begin{abstract}
In this paper a blind, imperceptible, robust and secure watermarking scheme for 3-D mesh models is presented. Here, the watermark is embedded in deeper surface vertices to minimize the perceivable distortion. Deeper surface vertices are selected on the basis of their mean curvature (lesser than zero) and converted to spherical coordinates. Out of the three spherical coordinates, radial distance represents approximate mesh and is invariant to distortionless attack. Therefore, watermark bits are embedded by modifying the distribution of radial distance to make the proposed scheme robust against such attacks. Radial distances are divided into bins and normalized to range in [0, 1]. Each bin accommodates one watermark bit. Watermark is embedded repeatedly in the 3-D mesh to resist cropping and simplification attack. To ensure higher security, a 128-bit unique watermark is generated by hashing (MD5 algorithm) the mean of histogram map obtained from a grayscale watermark image. Watermark bits are extracted from bins by comparing mean of each bin with a reference value. Since original mesh is not required at the time of extraction, the proposed scheme is blind. Through experimental results, it is demonstrate that the proposed scheme has good visual masking and higher robustness against various attacks. It shows improved performance as compared to some of the prominent schemes.
\end{abstract}

Keywords-3-D mesh watermarking; mean curvature; radial distance; spherical coordinates; visual masking

\section{INTRODUCTION}

In the past few decades there has been extensive growth in the use of 3-D objects in virtual reality, video games, animation, medical sciences, industries, and computer aided design (CAD), architecture, archaeology and scientific data visualization [1]. With the increasing accessibility and wide distribution of 3-D objects, the concern for its copyright protection has also increased. Digital watermarking is considered as a potential solution for copyright protection of various multimedia contents [2]. Therefore, researchers are exploring 3-D watermarking techniques for the copyright protection of 3-D mesh objects. Lot of research has been done in audio, image and video watermarking in spatial as well as transform domain. But, the watermarking schemes developed for other multimedia contents cannot be readily used for 3-D mesh due to lack of unique representation. 3-D objects are generally represented by polygonal meshes. Fig. 1 shows 3-D bunny model represented by triangular polygon mesh. 3-D mesh has three basic elements: vertices, edges, and faces [3]. Vertex is the co-ordinate in 3-D space. Edges are connectivity between two vertices. Face describes connectivity of vertices to form smallest unit of 3-D mesh. There is no well defined order and connectivity of vertices, which makes watermark extraction

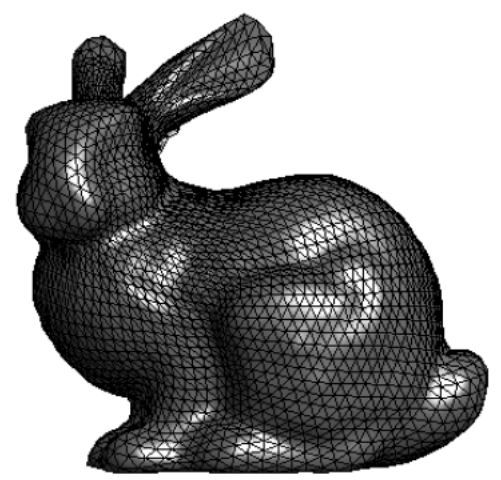

Fig. 1. 3-D Mesh of Bunny.

very difficult. Moreover, number of vertices where watermark bits can be inserted are limited as compared to other multimedia content. Due these challenges, 3-D mesh watermarking is still in its infancy [4].

There are different classifications for 3-D mesh watermarking techniques [5]. According to the insertion space it is classified into spatial and transform domain. In spatial domain, the watermark is inserted directly by altering the coordinates of the mesh vertices. But, in the transform domain watermark is inserted by altering the transform coefficients. Spatial techniques provide higher imperceptibility but are less robust. Some robust spatial watermarking schemes have been reported in [6]- [8]. But, the main drawback of these schemes is that they are relatively less robust to connectivity attacks. Whereas, transform domain techniques are robust but has higher computational time. Watermarking techniques can also be classified into non-blind and blind depending on whether the original host is required or not at the time of extraction. Non-blind schemes require original mesh during watermark extraction in contrast to blind schemes. Generally, non-blind techniques are more robust than blind techniques. Whereas blind schemes have high capacity, and can be easily implemented. But, practical applications prevent access to the original 3-D model during extraction. Considering pros and cons of different types of watermarking schemes, robust blind watermarking schemes in spatial domain are more feasible for practical applications. The majority of the exisiting 3D watermarking schemes suffers with low imperceptibility, robustness, security and high computational cost. For real time applications the computational cost is assumed to be low. Hence there is need to develop more blind and robust 
schemes for 3-D watermarking. In this paper a blind and robust 3-D watermarking scheme is presented which is robust to majority of attacks and maintains high imperceptibility. The rest of paper has following organization: Related work is reviewed in Section 2. The proposed 3-D watermarking scheme is described in detail in Section 3. Section 4 presents the experimental result and discussion. Section 5 concludes the paper.

\section{RELATED WORK}

Various 3-D mesh watermarking schemes have been proposed till date but still this field is in its infancy as compared to the development in image, audio and video watermarking. The two main hurdles in handling 3-D mesh watermarking are: lack of an ordered structure precludes systematic analysis of model and difficulty in anticipating all the attacks due to various mesh manipulation graphic tools. In spite of these challenges, researchers have put persistent efforts to propose various blind and non-blind 3-D mesh watermarking approaches for improving imperceptibility, robustness and payload.

In this direction, a blind scheme is proposed by Nakazawa et al. [9]. It uses mesh saliency based on the surface curvature to segment the triangular 3-D mesh. Then watermark bits are inserted in the regions by modulating the vertex norms. A similar approach is suggested by Zhan et al. [10] where watermark bits are interleaved in vertex bins formed on the basis of vertex curvature. Authors assert that this method has higher robustness and imperceptibility than the schemes proposed in [11], [12]. Li et al. [13] suggested to form bins of eigen values (distance between vertices to the model centre) based on improved vertex grouping. It uses piecewise mapping function to insert watermark bits in bins. It shows superior performance than [11]. Another blind scheme is proposed by Bata et al. [14] based on sparse QIM and run- length modulated LDPC. This scheme provides higher imperceptibility and lower BER in extracted watermark. Alaa et al. [15] proposed a blind scheme based on vertex distribution and skewness measure. Skewness measure is altered to embed the watermark. Authors claim that the scheme has good visual masking and resistant to a variety of common attacks. Recently, Sharma and Panda [7] have proposed a blind scheme and attempted to fulfil all the watermarking requirements by exploring Local curvature estimation and statistical characteristics for hiding watermark. Medimegh et al. [16] proposed a statistical 3-D watermarking scheme explored invariance property of salient points. They embedded statistical signature at different regions self-segmented around salient points. Hamid et al. [17] have proposed to a blind watermarking method in transform domain, where watermark is embedded in quantified wavelet coefficients using QIM based on 3-D mesh saliency. Though the scheme achieves good imperceptibility and robustness but, it is not robust against cropping attack. Other blind schemes has been reported in [18]- [21]. Some of the non-blind watermarking schemes are proposed in [22]- [25]. Although non-blind schemes exhibit satisfactory robustness but, mostly practical applications prevents having access to the original 3D model during extraction. Thus, blind schemes are practically more feasible. From the literature review it is observed that the existing schemes are robust to either one or the other set of attacks. Majority of existing schemes have focused on achieving higher imperceptibility and robustness but have overlooked an important watermarking characteristics i.e. security. A comprehensive comparision of the related wok is shown in Table I.

Motivation and contribution of the proposed work From the literature survey, we have observed that there is prospect to develop blind 3D watermarking scheme which is robust to majority of attacks, has higher imperceptibility and security. For practical application of the scheme it should be computationally inexpensive. Motivated by these issues we propose a 3-D watermarking scheme having following contribution:

- Improved robustness: the proposed blind scheme withstands majority of common attacks like smoothing, additive noise, similarity transformation, quantization, cropping and simplification as evident from experimental results. It successfully survives Meshbenchmark attacks. The proposed scheme has higher robustness as compared to the existing popular schemes in [10], [13], [17]. Although robustness of the proposed scheme against noise attack is not at par with [10], [13], [17] scheme but has acceptable correlation values as discussed in section IV.

- Improved visual quality: watermark embedding in deeper vertices of 3-D mesh surface induces less perceivable distortion than the vertices of flat or peak surface [26]. Making use of this observation, in the proposed scheme we select deeper surface vertices for embedding watermark bits to minimize induced distortion. Deeper surface vertices are classified on the basis of mean curvature $\left(C_{m}\right)$ [26]. Proposed scheme offers superior visual masking effect than schemes proposed in [10], [13], [17].

- Enhanced security: To enhance the watermark security, a 128-bit binary chaotic watermark sequnce is embedded in the 3-D model. This sequence is generated from a grayscale watermark image/logo. Mean of histogram map of the grayscale watermark image is calculated. Then the mean is hashed by using MD5 hash algorithm to obtain the watermark sequence. Watermark sequence is highly sensitive to the change in watermark image pixels, thus ensures high watermark security.

- Low computational cost: The proposed scheme embeds in spatial domain therefore it is less computationally expensive. The proposed embedding scheme has linear time complexity as discussed further in section IV.C.

\section{PRoposed WATERMARKING Scheme}

The proposed 3-D mesh watermarking scheme is based on modulation of radial distance distribution. Proposed scheme consists of three modules i.e. watermark generation, embedding and extraction, explained in detail in the following subsections. 
TABLE I. Comparative Study of 3-D Watermarking Schemes(SM-SMOOTHING, SP-SimPlification, SD-SUbdivision, CP-CROPPING)

\begin{tabular}{|c|c|c|c|c|c|c|c|c|}
\hline \multirow{3}{*}{ Method } & \multirow{3}{*}{ Domain } & \multicolumn{5}{|c|}{ Robustness against attacks } & \multirow{3}{*}{ Blind } & \multirow{3}{*}{ Capacity } \\
\hline & & \multicolumn{2}{|c|}{ Geometric } & \multicolumn{3}{|c|}{ Connective } & & \\
\hline & & SM & Noise & SP & SD & $\mathrm{CP}$ & & \\
\hline Jing et al. [24] & Spatial & Yes & Yes & $<5 \%$ & No & Yes & No & - \\
\hline Song $\mathrm{Li}$ et al. [13] & Spatial & Yes & Yes & NO & Yes & No & Yes & 64 bits \\
\hline Cho et al. [11] & Spatial & No & yes & $\leq 90 \%$ & Yes & No & yes & 64 bits \\
\hline $\begin{array}{l}\text { Garg and Agrawal } \\
{[22]}\end{array}$ & Spatial & No & Yes & $<70 \%$ & Yes & No & No & 256 bits \\
\hline Seung et. al. [19] & Spatial & Yes & Yes & $\leq 30 \%$ & No & Yes & Yes & 16 bits \\
\hline Alaa et. al. [15] & Spatial & No & Yes & $\leq 70 \%$ & No & Yes & Yes & 32 bits \\
\hline Zhan et al. [10] & Spatial & Yes & yes & $\leq 90 \%$ & No & No & Yes & Variable \\
\hline Hamidi [17] & Spectral & Yes & Yes & $\leq 70 \%$ & No & No & Yes & 64 bits \\
\hline Feng et. al [18] & Spectral & Yes & Yes & $\overline{\text { No }}$ & No & Yes & Yes & 36 bits \\
\hline Proposed & Spatial & Yes & Yes & $\leq 50 \%$ & No & yes & Yes & 128 bits \\
\hline
\end{tabular}

\section{A. Watermark Generation}

In the proposed scheme, to ensure higher security a unique 128-bit binary chaotic watermark sequence is generated according to the grayscale watermark/logo image of size $M \times M$. Firstly, a histogram map (HM) of grayscale watermark is generated. Then the mean of HM is calculated which is further used to generate an intermediate key. The resulting key is hashed by the hash function MD5 to obtain a 128-bit random binary sequence. This sequence is embedded in the 3-D model. Other hash algorithms like SHA can also be used for hashing, but its computation time and security should be taken into account. Watermark sequence is highly secure as the secret key is highly sensitive to the minor alteration in the watermark pixels. The watermark sequence can resist brute force attack. During extraction, the authorised user can obtain the secrete key according to the valid watermark image/logo provided for validation. Following are the steps for watermark generation:

Step 1: Generate the histogram map from the grayscale watermark image using transform function as follows:

$$
H M=\sum_{c=1}^{M}\left(H i s t p w_{c} \| P w_{c}\right), \forall P w[0,255]
$$

where Histpw is the frequency of a pixel $p w$ in watermark image.

Step 2: Generate intermediate key (8-bits of binary key sequence) from the HM as:

Transformfunc $=\sum_{c=1}^{M} \operatorname{dec}\left(H M_{c}\right) \bmod 2 \times 2^{14}$

intermediatekey $=$ mean $($ Transformfun $)$

where $d e c$ is decimal equivalent of $H M$.

Step 3: The resulting intermediatekey (8-bits) is hashed by MD5 hash function. A 128-bits binary sequence $(W)$ is generated by hashing. $W$ is used as a binary watermark sequence for embedding.

\section{B. Watermark Embedding}

Proposed watermark embedding scheme is based on the modulation of radial distance $(\rho)$ distribution in the deeper surface of mesh to reduce perceivable distortion and achieve higher robustness. Deeper surface vertices are selected on the basis of $C_{m}$. Vertices with $C_{m}<0$ are deeper vertices. Fig.2 shows grayscale representation of $C_{m}$ for bunny model, the darker area represents deeper vertices $\left(C_{m}<0\right)$ used for inserting the watermark, and the lighter area shows superficial vertices $\left(C_{m}>0\right)$.

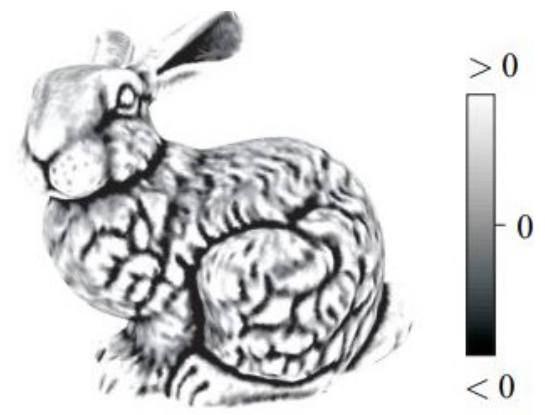

Fig. 2. Grayscale Representation of Mean Curvature in Bunny 3-D Model.

Selected vertices are converted from Cartesian coordinate to spherical coordinate $(\rho, \theta$ and $\varphi)$, shown in Fig. 3. Watermark is embedded by modulating $\rho_{i}$ as it is invariant to similarity and reordering transforms and represents approximately the mesh shape. Embedding in spherical coordinate particularly in $\rho$ is more robust than coordinates $(x, y, z)$. Radial distances of selected vertices are divided into bins and normalized to range $[0,1]$. One watermark bit is embedded in each bin by modulating radial distance using histogram mapping function as suggested in [11]. Watermark is embedded repeatedly to sustain attacks like simplification and cropping attack. Fig. 4 shows the block diagram of embedding process. Detailed steps for watermark embedding process are provided below.

\section{Steps of watermark embedding process}

\section{Step 1: Vertex selection}

Deeper surface vertices are selected for embedding having mean curvature less than zero. Mean curvature $\left(C_{m}\right)$ of vertices are calculated using method suggested by M. Mayer et al. [27]. $C_{m}$ is calculated using following equation.

$$
C_{m}=\left(\sum_{i=1}^{n} \theta\left(v_{i, j}\right)\right) / \frac{1}{3} \sum_{k=1}^{n 2} A_{k}
$$

$\theta\left(v_{i, j}\right)$ denotes the angles between the normal of the two adjacent triangles, and $A_{k}(\mathrm{k}=1,2, \ldots, \mathrm{n} 2)$ 


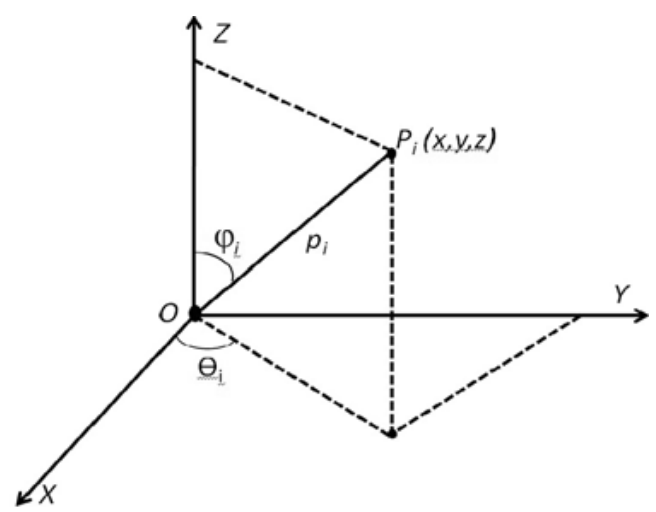

Fig. 3. Spherical Coordinates.

denotes the triangle area corresponding to the 1ring neighborhood of vertex. Vertex having $C_{m}<$ 0 are selected for embedding.

Step 2: Convert Cartesian coordinates to spherical coordinates

Cartesian coordinates of selected vertices $v_{i}$ are converted into spherical coordinates $\left(\rho_{i}, \theta_{i}\right.$ and $\varphi_{i}$ ) using given equations:

$$
\begin{gathered}
\rho_{i}=\left(\left(x_{i}-x_{g}\right)^{2}+\left(y_{i}-y_{g}\right)^{2}+\left(z_{i}-z_{g}\right)^{2}\right)^{1 / 2} \\
\theta_{i}=\tan ^{-1}\left(\left(y_{i}-y_{g}\right)^{2} /\left(x_{i}-x_{g}\right)^{2}\right) \\
\varphi_{i}=\cos ^{-1}\left(\left(z_{i}-z_{g}\right) / \rho_{i}\right)
\end{gathered}
$$

where $i$ is the $i^{t h}$ selected vertex and $\left(x_{g}, y_{g}, z_{g}\right)$ are the mean value of all vertices of 3-D model. For the proposed embedding scheme only radial distance $\left(\rho_{i}\right)$ of selected vertices are modified while keeping the other two coordinates i.e. $\varphi_{i}$ and $\theta_{i}$ unaltered.

Step 3: Bin formation

Bins are formed by dividing $\rho_{i}$ having equal range with respect to their values. One watermark bit is embedded in each bin. As watermark is embedded repeatedly, number of bins $\mathrm{N}$ is according to the watermark length $\mathrm{L}$ and number of repetitions. Bins are divided by using following equations:

$$
\begin{aligned}
B_{n} & =\left\{\rho_{n, j} \mid \rho_{\min }\right. \\
& +\left(\left(\rho_{\max }-\rho_{\min }\right) / N\right) \cdot n<\rho_{i}<\rho_{\min } \\
& \left.+\left(\left(\rho_{\max }-\rho_{\min }\right) / N\right) .(n+1)\right\}
\end{aligned}
$$

where $0 \leq \mathrm{n} \leq \mathrm{N}-1,0 \leq \mathrm{i} \leq$ number of selected vertices, $0 \leq \mathrm{j} \leq M_{n}-1, M_{n}$ is the number of radial distance of $n^{t h}$ bin and $\rho_{n, j}$ is the $j^{t h}$ radial distance of $n^{t h}$ bin. $\rho_{\max }$ and $\rho_{\min }$ are the maximum and minimum radial distance of the selected vertices in the mesh model.

Step 4: Bin normalization

Radial distances in the $n^{t h}$ bin are normalized in the range of $[0,1]$ using following equation:

$$
\begin{aligned}
\rho_{n, j} & =\left(\rho_{n, j}-\min _{\rho_{n, j} \epsilon B_{n}}\left\{\rho_{n, j}\right\}\right) \\
& \quad /\left(\max _{\rho_{n, j} \epsilon B_{n}}\left\{\rho_{n, j}\right\}-\min _{\rho_{n, j} \epsilon B_{n}}\left\{\rho_{n, j}\right\}\right)
\end{aligned}
$$

where $\hat{\rho}_{\dot{n}, j}$ is the normalized value of $\rho_{n, j}$, maximum and minimum radial distance of $n^{\text {th }}$ bin is represented by $\max _{\rho_{n, j} \in B_{n}}\left\{\rho_{n, j}\right\}$ and $\min _{\rho_{n, j} \in B_{n}}\left\{\rho_{n, j}\right\}$ respectively.

Step 5: Watermark embedding

One watermark bit is inserted per bin by transforming the radial distances using histogram mapping function as suggested in [11]. The mapping function is defined as $\mathrm{Eq} 10$.

$$
Y=X^{\beta} \quad \text { for } \quad 0<\beta<\infty \quad \beta \epsilon \Re
$$

Where $\mathrm{Y}$ is the transformed variable and the parameter $\beta$ is a real value. Watermark bit '1', is embeded by transforming $\rho$ to shift the mean by a factor $\beta$ and for watermark bit ' 0 ' shift mean by factor $-\beta$. Mean of every bin $\mu_{n}$ is shifted by following:

$$
\mu_{n}= \begin{cases}\frac{1}{2}+\alpha & \text { if } W=1 \\ \frac{1}{2}-\alpha & \text { if } W=0\end{cases}
$$

where $\alpha$ is the watermarking strength to determine robustness and imperceptibility. Mean is shifted by transforming each radial distance using Eq. 10 . An iterative algorithm is used to determine the value of parameter $\beta$ such that the value of modified radial distance exists within the range otherwise it may lead to serious distortions. Algorithm 1. shows the iterative process for determining the value of $\beta$ and embed watermark bits.

Step 6: Inverse Normalization

Transformed values of every bin are converted back to original range using following equation:

$$
\begin{aligned}
\rho_{n, j}^{\prime \prime} & =\left(\rho _ { n , j } ^ { \prime } \left(\max _{\rho_{n, j} \epsilon B_{n}}\left\{\rho_{n, j}\right\}\right.\right. \\
& \left.-\min _{\rho_{n, j} \epsilon B_{n}}\left\{\rho_{n, j}\right\}\right) \\
& \left.+\min _{\rho_{n, j} \epsilon B_{n}}\left\{\rho_{n, j}\right\}\right)
\end{aligned}
$$

Step 7: Conversion of spherical coordinates Cartesian to coordinates:

After completing embedding process, arrange and combine all the bins and convert spherical coordinates to Cartesian coordinates using following relation:

$$
\begin{gathered}
x_{i}{ }^{\prime}=\rho_{i}{ }^{\prime} \cos \theta_{i} \sin \varphi_{i}+x_{g} \\
y_{i}{ }^{\prime}=\rho_{i}{ }^{\prime} \sin \theta_{i} \sin \varphi_{i}+y_{g} \\
z_{i}{ }^{\prime}=\rho_{i}{ }^{\prime} \cos \varphi_{i}+z_{g}
\end{gathered}
$$

where $\left(x_{i}{ }^{\prime}, y_{i}{ }^{\prime}, y_{i}{ }^{\prime}\right)$ is the vertex Cartesian coordinate of watermarked model and $\rho_{i}{ }^{\prime}$ is the watermarked radial distance. $\theta_{i}, \varphi_{i}$ and center of gravity are same as obtained from Eq. 6 and Eq. 7. Finally, we get the watermarked 3-D mesh.

\section{Watermark Extraction}

Watermark extraction is just reverse process of watermark embedding. Vertices of watermarked mesh are selected and converted from Cartesian to spherical coordinates in the same way as in the embedding process. Radial distance is divided into bins and normalized using Eq. 8 and Eq. 


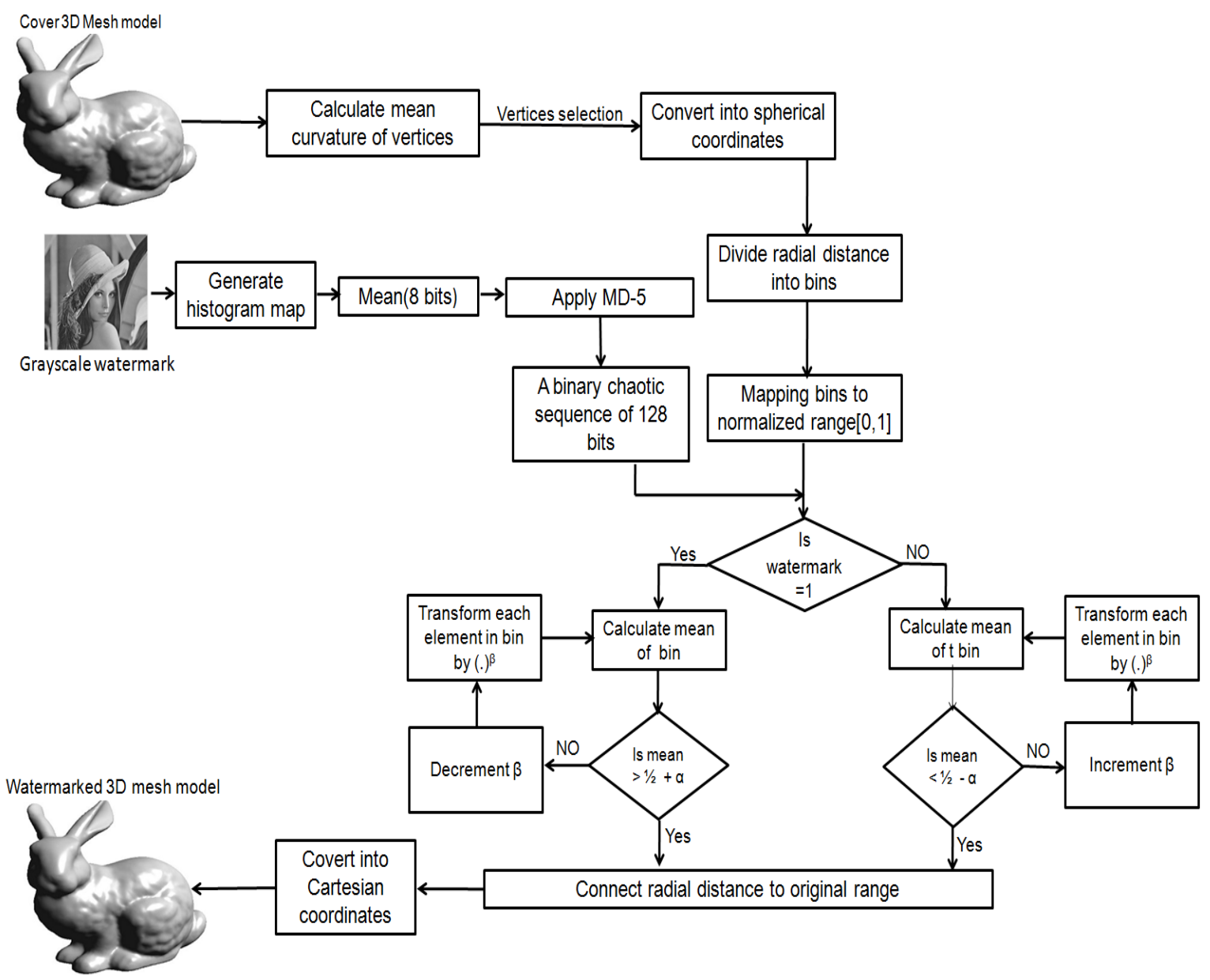

Fig. 4. Block Diagram of Embedding Process.

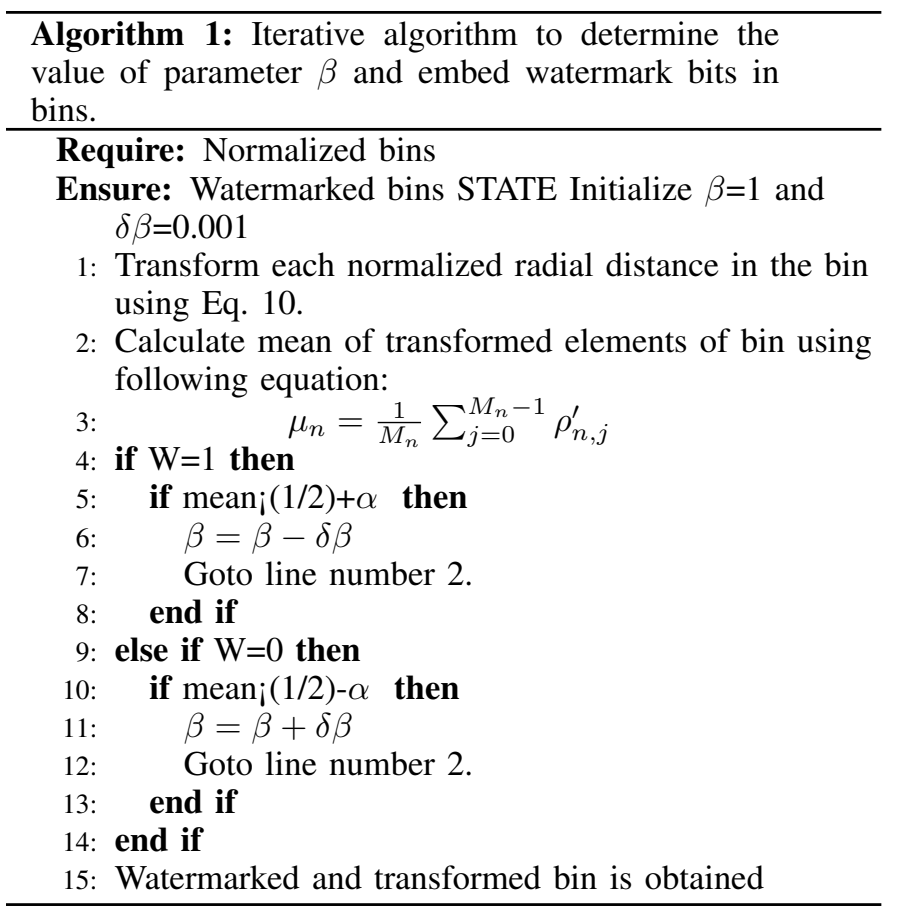

9. Then mean of each bin is compared with the reference value to extract the watermark. Original mesh is not required for extraction therefore extraction process is blind. More than one, independent watermark sequences are extracted from the bins. These watermark sequences are compared and according to the majority, the final watermark sequence is determined. It acts as error correction mechanism. For example, if $i^{\text {th }}$ bit of extracted watermarks has majority of 1 then the final watermark bit is 1 ; otherwise 0 . Finally, the extracted watermark $W^{\prime}$ is obtained. Fig. 5 shows the block diagram of extraction process. Steps for watermark extraction are explained below.

\section{Steps of watermark extraction process}

Step 1: Vertices are selected and converted into spherical coordinates as done in step 1 and 2 of watermark embedding process.

Step 2: Bins of radial distance are formed and normalized in the fashion similar to step 3 and 4 of embedding process.

Step 3: Mean is calculated for individual bin and compared with the reference value to extract watermark bits. One bit is extracted from each bin using 
following relation:

$$
w_{n}^{\prime}=\left\{\begin{array}{lll}
1 & \text { if } & \mu_{n}^{\prime}>1 / 2 \\
0 & \text { if } & \mu_{n}^{\prime}<1 / 2
\end{array}\right.
$$

Where $w_{n}^{\prime}$ is the extracted watermark bit from $n^{t h}, \mu_{n}^{\prime}$ is the mean of each bin.

Step 4: More than one watermark sequences are extracted. Watermark sequences are compared and the final watermark is decided on the basis of majority. Thus, we get 128-bit extracted watermark sequence $\left(W^{\top}\right)$.

\section{EXPERIMENTAL RESUlt AND Discussion}

This section presents experimental results to demonstrate the performance of proposed 3-D mesh watermarking scheme. Experiments were conducted to examine the robustness and imperceptibility of the proposed scheme using MATLAB on a PC with Intel core2 Duo, 3.00 GHz, 2GB RAM. Five different 3-D models i.e. Bunny, Armadillo, Dragon, Hand and Venus as shown in Fig. 6 were taken as test data set. These models represent variation of shapes and surfaces. Characteristics of 3-D test models are provided in Table II. Fig. 7 shows the watermarked mesh. For experimental evaluation, 128-bit binary watermark sequence, generated by the watermark generation process discussed in section 3.1 is used. The embedding capacity will vary for different 3-D mesh. This is due to lack of well-defined order and connectivity of vertices.

TABLE II. CharaCteristics OF 3-D MESh MODELS

\begin{tabular}{llllll}
\hline Object Model & Bunny & Armadillo & Dragon & Hand & Venus \\
\hline No. of vertices & 34835 & 30995 & 35000 & 36619 & 100759 \\
No. of faces & 69666 & 61986 & 70216 & 72958 & 201514 \\
\hline
\end{tabular}

\section{A. Imperceptibility (Visual Masking)}

Imperceptibility is one of the most important requirements for 3-D watermarking. Watermarking should cause minimum surface distortion so that it is not visible to human visual system (HVS). Subjective and objective analysis of visual quality of watermarked models is carried out. Subjective analysis is as important as objective analysis because sometimes the objective parameter may demonstrate good results but visual quality of 3-D object is not good. Mean opinion score (MOS) suggested by Corsini et al. [28] is used as metric for subjective analysis. Watermarked models are manually compared with original model from different angles. Observer assigns score between 0 and 10 to the watermarked mesh where 0 and 10 represents lowest and highest visual quality respectively. A normalized MOS is computed for each model by averaging the scores given by observers using following equation:

$$
M O S_{x}=\frac{1}{N} \sum_{y=1}^{N} S_{x y}
$$

Where $M O S_{x}$ is the mean opinion score of $x^{t h}$ model, $\mathrm{N}$ is the number of observer and $S_{x y}$ is the score for $x^{t h}$ model by $y^{\text {th }}$ observer.

Maximum root mean square error (MRMS) and Hausdorff distance(HD) are used as metric for objective analysis. MRMS
TABLE III. MRMS, MOS AND HAUSDORFF DISTANCE FOR WATERMARKED MODELS

\begin{tabular}{llllll}
\hline Object models & Bunny & Armadillo & Dragon & Hand & Venus \\
\hline MRMS $\left(\times 10^{3}\right)$ & 0.243 & 0.255 & 0.283 & 0.271 & 0.240 \\
MOS & 9.93 & 9.88 & 9.49 & 9.91 & 9.84 \\
Hausdorff Distance & 0.001 & 0.001 & 0.001 & 0.001 & 0.001 \\
\hline
\end{tabular}

measures surface-to-surface distance between the two meshes. Smaller MRMS value indicates better visual quality. MRMS is calculated by following equation:

$$
M R M S=\max \left(\operatorname{err}_{R M S}\left(M, M^{\prime}\right), \operatorname{err}_{R M S}\left(M^{\prime}, M\right)\right)
$$

where $\operatorname{err}_{R M S}$ is the root mean square error. $\operatorname{err}_{R M S}$ from one mesh $\mathrm{M}$ to another mesh $M^{\prime}$ is given by:

$$
\operatorname{err}_{R M S}\left(M, M^{\prime}\right)=(1 /|M|)^{1 / 2} \iint_{p \in M} d\left(p, M^{\prime}\right)^{2} d M
$$

where $\mathrm{p}$ is a point on the surface of mesh $\mathrm{M} .|M|$ is the area of $M$ and $d\left(p, M^{\prime}\right)$ is the point-to-surface distance between $p$ and $\mathrm{M}$.

$\mathrm{HD}$ is the maximum distance of a set to the nearest point in the other set of object. HD from set A to set B is a max-min function, defined as

$$
H D\left(O, O^{w}\right)=\max \{\min \{d(a, b)\}\}
$$

where, $\mathrm{a}$ and $\mathrm{b}$ are points of original set $\mathrm{O}$ and watermark object $O^{w}$ respectively, and $\mathrm{d}(\mathrm{a}, \mathrm{b})$ is any metric between these points.

To study the imperceptibility and distortion in watermarked model, both subjective and objective evaluation is done in terms of MRMS,MOS and HD and the results are tabulated in Table III. Subjectively analysis, of original and watermarked models reveals that there is no significant visual distortion is induced. Table III shows that MOS is close to 10 in all models which indicate that no significant perceivable distortion is caused by the proposed scheme. It can be observed from Table III that MRMS and HD values are also very low. Hence, the proposed scheme satisfies both the perceptual and the geometric quality in the 3-D Mesh watermarking.

\section{B. Robustness Evaluation}

Robustness is another important requirements of 3-D mesh watermarking scheme. Robustness of proposed scheme is evaluated under different common attacks like, smoothing attack, cropping attack, noise addition, quantization, similarity transformation, simplification attack and scaling on $\mathrm{Z}$ axis. Simulation of noise addition and scaling attack is done in MATLAB whereas smoothing, simplification and cropping attacks is simulated by Meshlab open source [28]. Also, the robustness of the proposed scheme is evaluated under attacks of Meshbenchmark tool. Correlation coefficient and BER is used to measure the robustness. It measures the similarity between embedded and extracted watermark and can be express as following equation: 


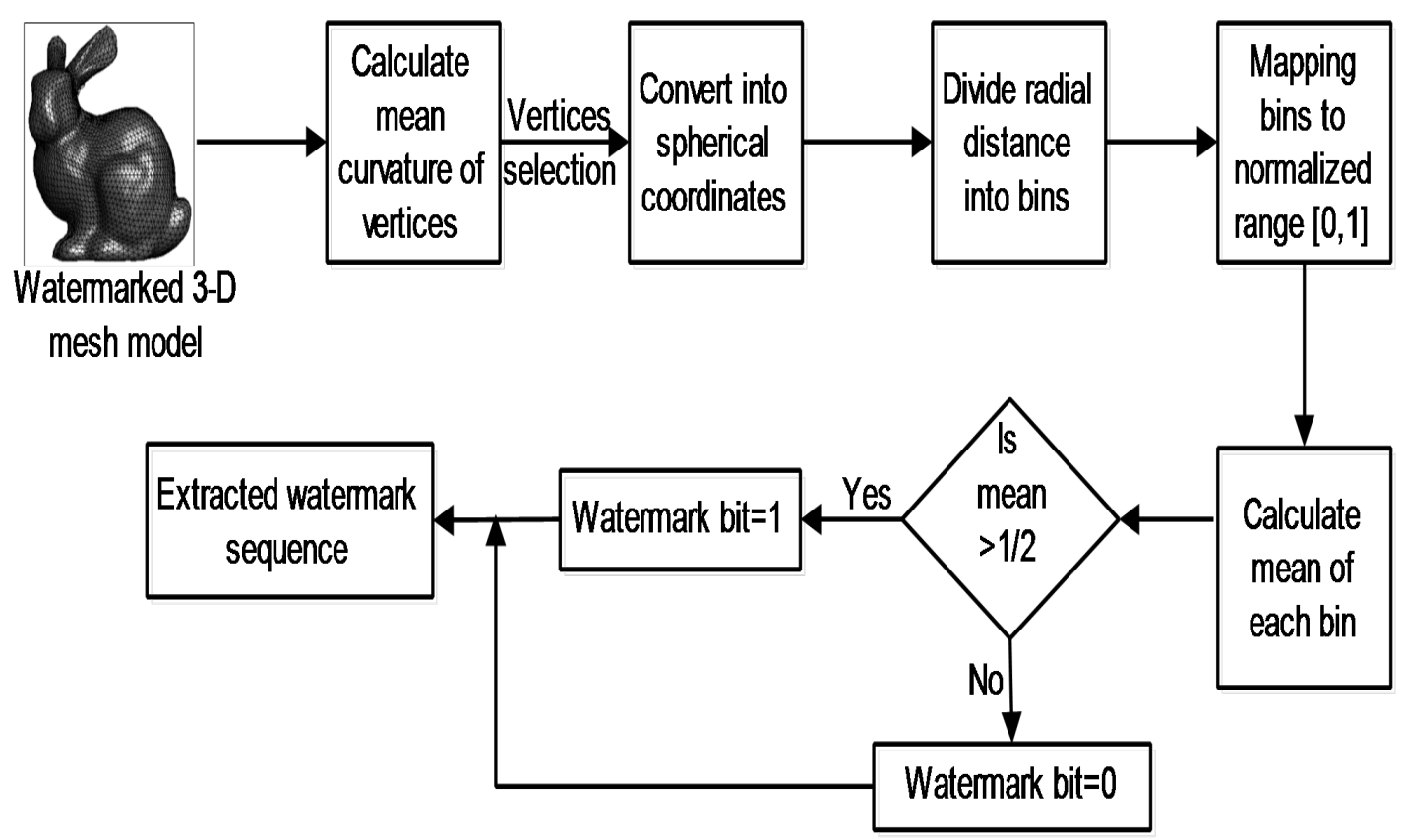

Fig. 5. Block Diagram of Extraction Process.

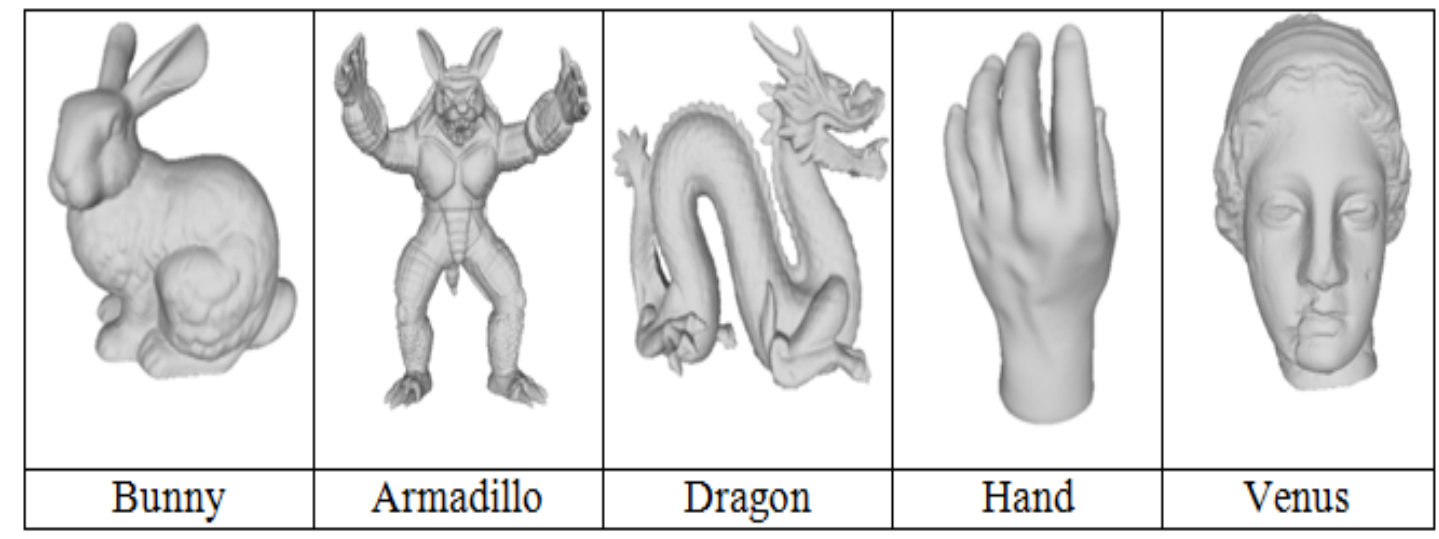

Fig. 6. Original 3-D Mesh Models.

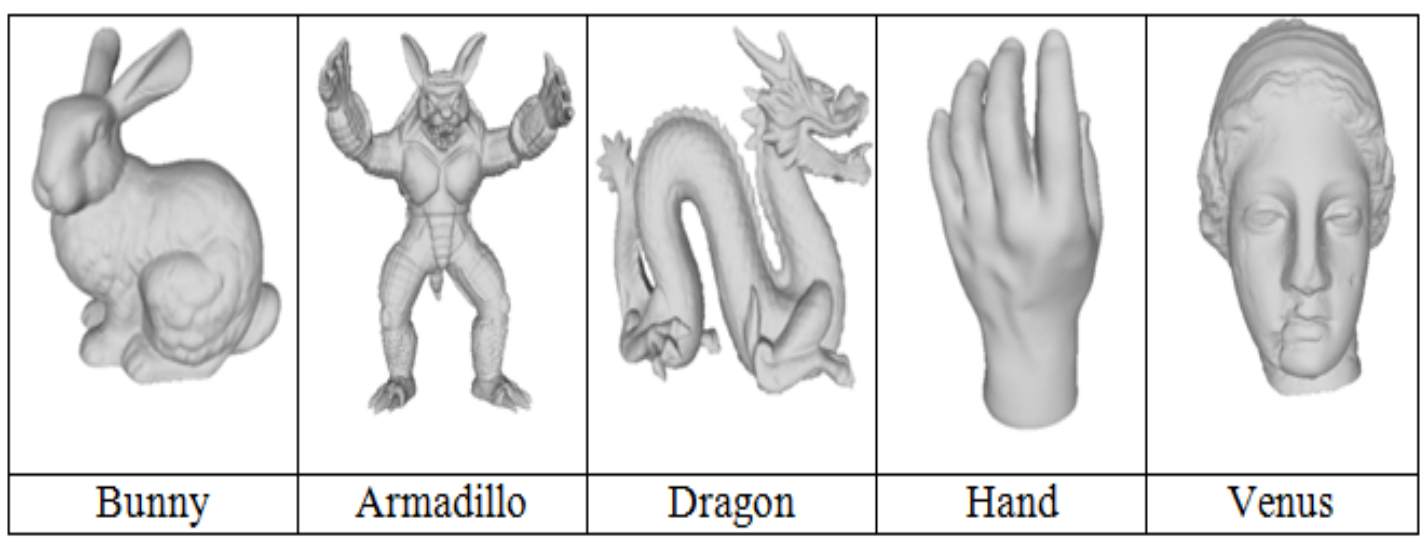

Fig. 7. Watermarked 3-D Mesh Models. 


$$
\begin{aligned}
\operatorname{Corr}\left(W, W^{\prime}\right) & =\sum_{i=0}^{L-1}\left(w_{i}^{\prime}-\bar{w}^{\prime}\right)\left(w_{i}-\bar{w}\right) \\
& /\left(\sum_{i=0}^{L-1}\left(w_{i}^{\prime}-\bar{w}^{\prime}\right)^{2} \sum_{i=0}^{L-1}\left(w_{i}-\bar{w}\right)^{2}\right)^{1 / 2}
\end{aligned}
$$

where $\mathrm{W}$ and $W^{\prime}$ are embedded and extracted watermark respectively. $\bar{w}$ and $\bar{w}^{\prime}$ are the mean of embedded and extracted watermark, $w_{i}$ and $w_{i}^{\prime}$ are the $i^{t h}$ watermark bit of embedded and extracted watermark, respectively. Correlation ranges in $[-1,1]$ and the ideal value is 1 .

When the watermark is extracted from watermarked model without any attack, the corr value is 0.9992 quite close to the ideal value. Watermark embedding process effects the correct bin division in the watermark extraction process therefore ideal value of corr could not be achieved.

1) Robustness Against Smoothing Attack: Smoothing is commonly used to eliminate the noise introduced during the mesh generation process. There are many smoothing algorithms out of which Laplacian smoothing algorithm proposed in [29] is commonly used. Therefore, in existing literature majority of the 3-D watermarking schemes test robustness of their scheme under Laplacian smoothing attack. It is an iterative process where vertex position is calculated by averaging its neighbours and the weight is determined according to its local connectivity. The results of Laplacian smoothing with different number of iteration ranging from 10-50 and keeping deformation factor $\lambda=0.03$ is presented in Table VI. From Table VI, it can be observed that the corr value for all models is appreciable upto 30 iterations. Also, the extracted watermark is distinguishable for less than 50 iterations as the normalized correlation is above the threshold value. Hence, the proposed scheme has fair robustness under smoothing attack.

2) Robustness Against Cropping: Cropping attack deletes vertices from one or more parts of a 3-D mesh model. It destroys the watermark information from the cropped region but watermark can be validated from the remaining portion. Cropping attack does not destroy all watermarked vertices because watermark bits are scattered uniformly. In the proposed scheme watermark is repetitively embedded to resist cropping attack. From the Table IV it can be observed that corr value for $10 \%, 20 \%$ and $25 \%$ cropping are more than $0.92,0.83$ and 0.75 indicating that extracted watermark is distinguishable. Thus, the proposed scheme is robust against cropping attack, because for all models corr value is more than the threshold.

TABLE IV. Corr VAlues For 3-D MOdels under CRopping ATtack

\begin{tabular}{llllll}
\hline Cropping \% & Bunny & Armadillo & Dragon & Hand & Venus \\
\hline 10 & 0.9235 & 0.9556 & 0.9375 & 0.9607 & 0.9451 \\
20 & 0.8371 & 0.8593 & 0.8299 & 0.8788 & 0.8488 \\
25 & 0.7598 & 0.7827 & 0.7621 & 0.7494 & 0.7399 \\
\hline
\end{tabular}

3) Robustness Against Simplification Attack: Mesh simplification is commonly used operation particularly for compression. A highly detailed mesh can be simplified or conversely increase the complexity of simplified mesh. Table $\mathrm{V}$ shows Corr values for varying simplification ratio. The proposed scheme shows satisfactory resistance towards simplification attack.

TABLE V. CORR VALUES FOR 3-D MOdELS UNDER SimplificATION ATTACK

\begin{tabular}{cccccc}
\hline $\begin{array}{c}\text { Simplification vertex } \\
\text { reduction ratio (\%) }\end{array}$ & Bunny & Armadillo & Dragon & Hand & Venus \\
\hline 70 & 0.9046 & 0.8571 & 0.8735 & 0.8579 & 0.8699 \\
90 & 0.8136 & 0.7889 & 0.8025 & 0.7792 & 0.7964 \\
\hline
\end{tabular}

TABLE VI. CORR VALUES FOR 3-D MODELS UNDER LAPLACIAN Smoothing Attack $(\lambda=0.03)$

\begin{tabular}{llllll}
\hline Iterations & Bunny & Armadillo & Dragon & Hand & Venus \\
\hline 10 & 0.9386 & 0.9025 & 0.9144 & 0.9247 & 0.9197 \\
30 & 0.8692 & 0.8703 & 0.8463 & 0.8836 & 0.8961 \\
50 & 0.6173 & 0.6899 & 0.5894 & 0.6391 & 0.5939 \\
\hline
\end{tabular}

4) Robustness Against Random Additive Noise: Noise attack is very common during data transmission and mesh object operation like read/write. Pseudo random noise (amplitude $\epsilon 0.05 \%, 0.25 \%, 0.5 \%)$ is added on vertex to distort the watermark. Experimental results of random additive noise attacks in Table VII shows that proposed scheme shows good robustness even at high noise ratio of $0.5 \%$.

TABLE VII. CORR VALUES For 3-D MOdels Under AdDitive NoISE ATTACK

\begin{tabular}{llllll}
\hline Noise \% & Bunny & Armadillo & Dragon & Hand & Venus \\
\hline 0.1 & 0.9883 & 0.9497 & 0.9578 & 0.9479 & 0.9397 \\
0.3 & 0.9146 & 0.8692 & 0.8749 & 0.8491 & 0.8739 \\
0.5 & 0.8139 & 0.7616 & 0.7509 & 0.7787 & 0.7989 \\
\hline
\end{tabular}

5) Robustness Against Quantization Attack : Quantization is lossy compression method. We have evaluated robustness of our scheme against different quantization rate. Vertex coordinate is represented with 7, 8 and 9 bits. Table VIII shows the corr values against different quantization attack. Proposed method is robust for 9-bit and 8-bit quantization attack.

TABLE VIII. CORR VALUES FOR 3-D MODELS UNDER ADDITIVE QUANTIZATION ATTACK

\begin{tabular}{llllll}
\hline Quantization \% & Bunny & Armadillo & Dragon & Hand & Venus \\
\hline 9 bits & 0.9983 & 0.9448 & 0.9753 & 0.9488 & 0.9961 \\
8 bits & 0.9461 & 0.9121 & 0.8942 & 0.8813 & 0.8498 \\
7 bits & 0.6191 & 0.7114 & 0.5529 & 0.6771 & 0.7389 \\
\hline
\end{tabular}

6) Robustness Against Similarity Transform: It is desireable for any 3-D watermarking scheme to be robust against similarity transform as it includes rotation, translation, and uniform scaling attack. Table IX presents corr values under similarity transformation. It can be observed from Table IX that the proposed watermarking scheme is robust against this attack for all 3-D model used for evaluation.

7) Robustness Against Scaling Attack on Z-axis : Uniform scaling attack on Z-axis having scaling factor 1.25 is applied on 3-D models. Results are tabulated in following Table X. Proposed scheme is fairly robust against scaling attack. 
TABLE IX. CORR VALUES FOR 3-D MODElS UNDER SiMILARITY TRANSFORMATION

\begin{tabular}{lclccc}
\hline $\begin{array}{l}\text { Similarity } \\
\text { Transformation }\end{array}$ & Bunny & Armadillo & Dragon & Hand & Venus \\
\hline Transformaton1 & 0.9826 & 0.9762 & 0.9635 & 0.9833 & 0.9587 \\
Transformaton2 & 0.9399 & 0.9527 & 0.9192 & 0.9384 & 0.9086 \\
Transformtion3 & 0.9188 & 0.9129 & 0.8973 & 0.9118 & 0.8893 \\
\hline
\end{tabular}

TABLE X. CORR VALUES FOR 3-D MODELS UNDER SCALing ATTACK

\begin{tabular}{llllll}
\hline Attacks & Bunny & Armadillo & Dragon & Hand & Venus \\
\hline Scaling on & 0.9921 & 0.9858 & 0.9824 & 0.9907 & 0.9843 \\
Z-axis (1.25) & & & & \\
\hline
\end{tabular}

8) Robustness Evaluation using MeshBenchmark Tool: Robustness of the proposed scheme has been evaluated using benchmark tool Meshbenchmark [30] available at http://liris.cnrs.fr/meshbenchmark/. Table XI shows BER values of extracted watermark under various attacks defined by perceptual and geometric quality oriented protocol of Meshbenchmark. From Table XI we observe that the proposed method is more suitable for the applications where the watermarked object of high visual quality is needed.

\section{Time Complexity}

In this subsection we analyze the asymptotic time complexity of the proposed embedding scheme. While calculating the computational cost we consider the cost of major algorithmic steps for watermark embedding and the most expensive operations only. Computationally expensive operations are as follows:

- Vertex selection: As specified earlier vertices with mean curvature less than zero are selected for embedding. Therefore mean curvature for each vertex is calculated using Eq. 4. Computational cost incurred in 1-ring neighborhood construction by traversing each edge is $O(3 \mathrm{~V})$ [31]. Therefore computational cost is $O(3 \mathrm{~V})$.

- Conversion of Cartesian coordinate to spherical coordinate: Each selected vertex is converted from Cartesian to spherical coordinate. Computational cost of conversion is $O(V)$.

- $\quad$ Determination of $\beta$ and watermark embedding: Value of $\beta$ is determined by an iterative algorithm.

TABLE XI. BER Values under Various Meshbenchmark ATtacks

\begin{tabular}{lcc}
\hline Attacks & BER (Perceptual) & BER (Geometric) \\
\hline Similarity transformation & 0.01 & 0.01 \\
Noise A $=0.10 \%$ & 0.01 & 0.04 \\
Noise A $=0.30 \%$ & 0.08 & 0.12 \\
Noise $\mathrm{A}=0.50 \%$ & 0.19 & 0.25 \\
Smoothing $N_{\text {itr }}=10$ & 0.13 & 0.22 \\
Smoothing $N_{\text {itr }}=30$ & 0.29 & 0.37 \\
Smoothing $N_{i t r}=50$ & 0.39 & 0.42 \\
Quantization $\mathrm{R}=9$ & 0.09 & 0.16 \\
Quantization $\mathrm{R}=8$ & 0.18 & 0.21 \\
Quantization $\mathrm{R}=7$ & 0.42 & 0.51 \\
Simplification $E_{\text {sim }}=10 \%$ & 0.01 & 0.01 \\
Simplification $E_{\text {sim }}=30 \%$ & 0.02 & 0.03 \\
Simplification $E_{\text {sim }}=50 \%$ & 0.06 & 0.17 \\
Cropping $V_{c r}=10 \%$ & 0.51 & 0.53 \\
Cropping $V_{c r}=30 \%$ & 0.55 & 0.56 \\
Subdivision Midpoint & 0.01 & 0.03 \\
\hline
\end{tabular}

The computational cost is $O(n V)$ where $\mathrm{n}$ is the number of iterations.

Based on the above analysis, we conclude that the proposed watermarking scheme has linear time complexity as:

$$
O(3 V)+O(V)+O(n V)=O(n V)
$$

TABLE XII. AVERAge Number of Iterations AND COMPUTATION TIME FOR DETERMING $\beta$ PER BIN

\begin{tabular}{lcc}
\hline Model & Avg. number of iterations & Avg. computation time(ms) \\
\hline Bunny & 154 & 13.09 \\
Armadillo & 358 & 235.16 \\
Dragon & 205 & 121.83 \\
Hand & 139 & 81.48 \\
Venus & 742 & 533.64 \\
\hline
\end{tabular}

The average number of iteration and computation time required for determining $\beta$ for each bin is presented in Table XII. The total time required for watermark embedding ranges from $0.6 \mathrm{~s}$ to $200 \mathrm{~s}$ subjected to mesh size.

\section{Comparative Analysis}

To further validate the performance of the proposed scheme, a comparative study is done with existing 3-D mesh watermarking schemes in terms of imperceptibility, robustness and security. Comparison has been done with spatial domain schemes proposed by Zhan et al. [10], Li et al. [13], and transform domain scheme proposed by Hamidi et al. [17]. In the scheme proposed by Zhan et al. [10], watermark bits are interleaved in bins formed on the basis of vertex curvature. Similarly, Li et al. [13] suggested to form bins of eigen values (distance between vertice to the model center ) based on improved vertex grouping. It uses piecewise mapping function to insert watermark bits in the bins. Both the watermarking schemes are in spatial domain and blind. They show improved imperceptibility and robustness than [11], but none of the them have taken security requirement of watermarking in account. Hamid et al. [17] have proposed to a blind watermarking scheme in transform domain, where watermark is embedded in quantified wavelet coefficients using QIM based on 3-D mesh saliency. Though the scheme achieves good imperceptibility and robustness but, it is not able to resist cropping attack. They have used key, but its effectiveness in providing security is debatable. Schemes in comparison, assert to have better performance than the state-of-the-art schemes in exsisting litreature.

Imperceptibility of the proposed scheme is compared with the other counterparts [10], [13], [17] in terms of MRMS value. Fig. 8 shows the MRMS value for three watermarked 3-D mesh (without attack) i.e. Bunny, Dragon and Venus. MRMS value of the proposed scheme is lower than [10], [17] and quite equal to [13]. Hence, it can be inferenced that the proposed scheme outperforms [10], [17] and is at par with [13] in terms of imperceptibility. Higher imperceptibility of the proposed scheme can be attributed to the embedding of watermark bits in deeper vertices.

Further, comparison of performance under different attacks is studied in terms of Corr and MRMS value. Table XIII shows comparison of robustness and imperceptibility of the proposed scheme with Zhan et al. scheme [10]. It can be observed from 
TABLE XIII. COMPARISON OF CORR AND MRMS VALUES OF PROPOSED SCHEME With ZHAN ET AL. SCHEME [10]

\begin{tabular}{|c|c|c|c|c|c|c|}
\hline \multirow{2}{*}{ Models } & \multirow{2}{*}{ Attacks } & \multirow{2}{*}{ Parameters } & \multicolumn{2}{|c|}{ Proposed Scheme } & \multicolumn{2}{|c|}{ Zhan et al. [10] } \\
\hline & & & Corr & MRMS & Corr & MRMS \\
\hline \multirow{12}{*}{ Bunny } & \multirow{3}{*}{ Smoothing } & $N_{\text {iter }}=10$ & 0.9386 & 0.18 & 0.92 & 0.26 \\
\hline & & $N_{\text {iter }}=30$ & 0.8692 & 0.53 & 0.85 & 0.70 \\
\hline & & $N_{\text {iter }}=50$ & 0.6173 & 0.64 & 0.44 & 0.97 \\
\hline & \multirow{3}{*}{ Quantization } & 9-bit & 0.9968 & 0.49 & 1.00 & 0.61 \\
\hline & & 8-bit & 0.9562 & 0.88 & 0.91 & 1.00 \\
\hline & & 7-bit & 0.7311 & 1.12 & 0.58 & 1.84 \\
\hline & \multirow{2}{*}{ Simplification } & $70 \%$ & 0.9046 & 0.23 & 1.00 & 0.40 \\
\hline & & $90 \%$ & 0.7936 & 0.41 & 0.83 & 0.54 \\
\hline & \multirow{3}{*}{ Noise } & $0.1 \%$ & 0.9883 & 0.27 & 1.00 & 0.32 \\
\hline & & $0.3 \%$ & 0.8463 & 0.36 & 0.91 & 0.40 \\
\hline & & $0.5 \%$ & 0.8139 & 0.59 & 0.80 & 0.98 \\
\hline & Cropping & $25 \%$ & 0.7598 & 0.81 & 0.73 & 1.02 \\
\hline \multirow{12}{*}{ Dragon } & \multirow{3}{*}{ Smoothing } & $N_{\text {iter }}=10$ & 0.9144 & 0.28 & 0.91 & 0.33 \\
\hline & & $N_{\text {iter }}=30$ & 0.8463 & 0.79 & 0.66 & 0.80 \\
\hline & & $N_{\text {iter }}=50$ & 0.5894 & 0.91 & 0.44 & 1.12 \\
\hline & \multirow{3}{*}{ Quantization } & 9-bit & 0.9782 & 0.48 & 0.96 & 0.55 \\
\hline & & 8-bit & 0.7911 & 0.86 & 0.77 & 1.13 \\
\hline & & 7-bit & 0.5681 & 0.95 & 0.41 & 1.89 \\
\hline & \multirow{2}{*}{ Simplifcation } & $70 \%$ & 0.8735 & 0.31 & 0.95 & 0.33 \\
\hline & & $90 \%$ & 0.8025 & 0.22 & 0.66 & 0.97 \\
\hline & \multirow{3}{*}{ Noise } & $0.1 \%$ & 0.9578 & 0.33 & 1.00 & 0.32 \\
\hline & & $0.3 \%$ & 0.8692 & 0.59 & 0.91 & 0.62 \\
\hline & & $0.5 \%$ & 0.7609 & 0.93 & 0.77 & 1.01 \\
\hline & Cropping & $25 \%$ & 0.7827 & 0.95 & 0.77 & 1.01 \\
\hline \multirow{12}{*}{ Venus } & \multirow{3}{*}{ Smoothing } & $N_{i t e r}=10$ & 0.9197 & 0.28 & 1.00 & 0.12 \\
\hline & & $N_{\text {iter }}=30$ & 0.8961 & 0.31 & 0.97 & 0.24 \\
\hline & & $N_{i t e r}=50$ & 0.5939 & 0.49 & 0.93 & 0.59 \\
\hline & \multirow{3}{*}{ Quantization } & 9-bit & 0.9963 & 0.52 & 1.00 & 0.64 \\
\hline & & 8 -bt & 0.9485 & 0.93 & 0.83 & 1.12 \\
\hline & & 7-bit & 0.8428 & 1.02 & 0.73 & 1.75 \\
\hline & \multirow{2}{*}{ Simplification } & $70 \%$ & 0.8699 & 0.24 & 1.00 & 0.30 \\
\hline & & $90 \%$ & 0.7964 & 0.33 & 0.96 & 0.32 \\
\hline & \multirow{3}{*}{ Noise } & $0.1 \%$ & 0.9397 & 0.27 & 0.95 & 0.24 \\
\hline & & $0.3 \%$ & 0.8739 & 0.48 & 0.95 & 0.58 \\
\hline & & $0.5 \%$ & 0.7989 & 1.01 & 0.79 & 0.99 \\
\hline & Cropping & $25 \%$ & 0.7494 & 0.89 & 0.72 & 0.96 \\
\hline
\end{tabular}

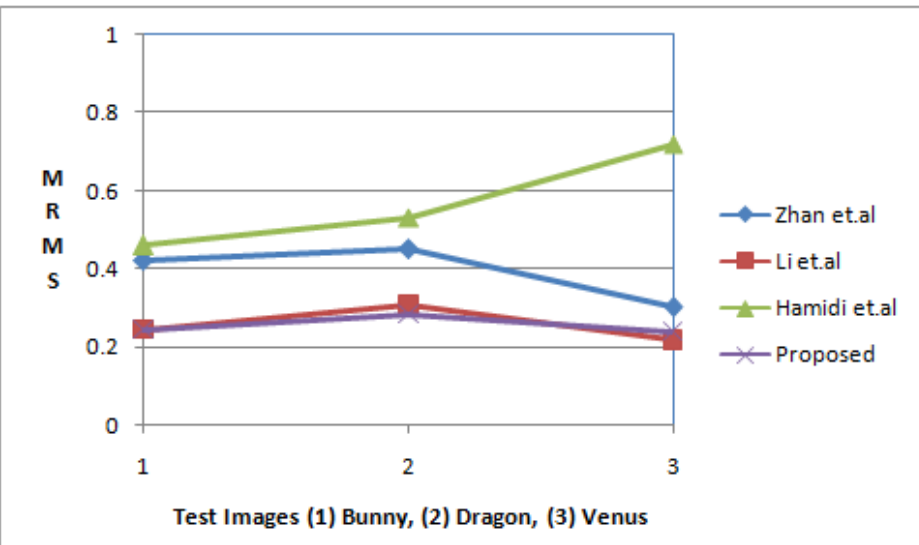

Fig. 8. MRMS Value for Watermarked Bunny, Dragon and Venus for Schemes Proposed in [10], [13], [17].

Table XIII that the proposed scheme has higher robustness and imperceptibility under smoothing, quantization and cropping attack for majority of models in comparison. In simplification attack, correlation value of the proposed scheme is lesser than [10] but higher than the threshold. Under these attacks, MRMS values are comparitively higher for the proposed scheme indicating higher imperceptibility. And, the proposed scheme has vying performance against simplification, noise and scaling attack. Therefore, it can be said that the proposed scheme outperforms in most of the common attacks than Zhan et
TABLE XIV. COMPARISON OF CORR AND MRMS VALUES OF PROPOSED SCHEME WITH LI ET AL. SCHEME [13] UNDER DifFERENT ATTACKS

\begin{tabular}{|c|c|c|c|c|c|c|}
\hline \multirow{2}{*}{ Models } & \multirow{2}{*}{ Attacks } & \multirow{2}{*}{ Parameters } & \multicolumn{2}{|c|}{ Proposed Scheme } & \multicolumn{2}{|c|}{ Song $\mathrm{Li}$ et al. $[13]$} \\
\hline & & & Corr & MRMS & Corr & MRMS \\
\hline \multirow{9}{*}{ Bunny } & \multirow{3}{*}{ Smoothing } & $N_{\text {iter }}=10$ & 0.9386 & 0.18 & 1.00 & 0.342 \\
\hline & & $N_{\text {iter }}=30$ & 0.8692 & 0.53 & 0.77 & 0.705 \\
\hline & & $N_{\text {iter }}=50$ & 0.6173 & 0.64 & 0.38 & 1.046 \\
\hline & \multirow{3}{*}{ Quantization } & 9-bit & 0.9968 & 0.49 & 0.97 & 0.528 \\
\hline & & 8-bit & 0.9562 & 0.88 & 0.58 & 1.076 \\
\hline & & 7-bit & 0.7311 & 1.12 & 0.17 & 2.085 \\
\hline & \multirow{3}{*}{ Noise } & $0.1 \%$ & 0.9883 & 0.27 & 1.00 & 0.327 \\
\hline & & $0.3 \%$ & 0.8463 & 0.36 & 0.90 & 0.712 \\
\hline & & $0.5 \%$ & 0.8139 & 0.59 & 0.68 & 1.141 \\
\hline \multirow{9}{*}{ Dragon } & \multirow{3}{*}{ Smoothing } & $N_{\text {iter }}=10$ & 0.9144 & 0.28 & 1.00 & 0.412 \\
\hline & & $N_{\text {iter }}=30$ & 0.8463 & 0.79 & 0.80 & 0.856 \\
\hline & & $N_{\text {iter }}=50$ & 0.5894 & 0.91 & 0.55 & 1.301 \\
\hline & \multirow{3}{*}{ Quantization } & 9-bit & 0.9782 & 0.48 & 0.97 & 0.643 \\
\hline & & 8-bit & 0.7911 & 0.86 & 0.52 & 1.181 \\
\hline & & 7-bit & 0.5681 & 0.95 & 0.25 & 2.319 \\
\hline & \multirow{3}{*}{ Noise } & $0.1 \%$ & 0.9578 & 0.33 & 1.00 & 0.391 \\
\hline & & $0.3 \%$ & 0.8692 & 0.59 & 1.00 & 0.795 \\
\hline & & $0.5 \%$ & 0.7609 & 0.93 & 0.97 & 1.255 \\
\hline \multirow{9}{*}{ Venus } & \multirow{3}{*}{ Smoothing } & $N_{\text {iter }}=10$ & 0.9197 & 0.28 & 1.00 & 0.238 \\
\hline & & $N_{\text {iter }}=30$ & 0.8961 & 0.31 & 0.94 & 0.379 \\
\hline & & $N_{\text {iter }}=50$ & 0.5939 & 0.49 & 0.72 & 0.535 \\
\hline & \multirow{3}{*}{ Quantization } & 9-bit & 0.9963 & 0.52 & 0.88 & 0.697 \\
\hline & & 8 -bt & 0.9485 & 0.93 & 0.58 & 1.340 \\
\hline & & 7-bit & 0.8428 & 1.02 & -0.12 & 2.704 \\
\hline & \multirow{3}{*}{ Noise } & $0.1 \%$ & 0.9397 & 0.27 & 1.00 & 0.391 \\
\hline & & $0.3 \%$ & 0.8739 & 0.48 & 0.72 & 1.006 \\
\hline & & $0.5 \%$ & 0.7989 & 1.01 & 0.25 & 1.654 \\
\hline
\end{tabular}

TABLE XV. COMParison of CORR VALues of Proposed SCHEME AND HAMIDI ET AL. SCHEME [17] FOR BUNNY

\begin{tabular}{cccc}
\hline \multirow{2}{*}{ Attacks } & \multirow{2}{*}{ Parameters } & Proposed & Hamidi et al. [17] \\
\cline { 2 - 4 } & & Corr & Corr \\
\hline \multirow{3}{*}{ Smoothing } & $N_{\text {iter }}=10$ & $\mathbf{0 . 9 3 8 6}$ & 0.92 \\
& $N_{\text {iter }}=30$ & $\mathbf{0 . 8 6 9 2}$ & 0.88 \\
& $N_{\text {iter }}=50$ & 0.6173 & 0.71 \\
\hline \multirow{3}{*}{ Quantization } & $9-$ bit & $\mathbf{0 . 9 9 6 8}$ & 0.99 \\
& 8-bit & $\mathbf{0 . 9 5 6 2}$ & 0.95 \\
& 7-bit & 0.7311 & 0.89 \\
\hline \multirow{2}{*}{ Noise } & $0.1 \%$ & 0.9883 & 0.99 \\
& $0.3 \%$ & 0.8463 & 0.90 \\
\hline \multirow{2}{*}{ Simplification } & $0.5 \%$ & 0.8139 & 0.84 \\
\hline Cropping & $70 \%$ & $\mathbf{0 . 9 0 4 6}$ & 0.71 \\
& $90 \%$ & $\mathbf{0 . 7 9 3 6}$ & 0.46 \\
\hline
\end{tabular}

al. scheme [10]. Similarly, performance comparision of the proposed scheme has been done with $\mathrm{Li}$ et al. scheme [13] in Table XIV. It can be observed from Table XIV that the proposed scheme outperforms [13] in terms of imperceptibility under all attacks. The proposed scheme performs better under smoothing and quantization attacks. Though, the robustness of the Li et al. scheme [13] is higher under noise attack than the proposed scheme but lags behind in imperceptibility. Thus, we can deduce that the proposed scheme outdo [13]. Performance of the proposed scheme is also compared with Hamidi et al. scheme [17] under different attacks for Bunny model and the results have been tabulated in Table XV. It can be observed that the proposed scheme is more robust than Hamidi et al. scheme [17] under common attacks except noise attack.

An overall comparison of robustness for the proposed scheme and counterpart schemes [10], [13], [17] under smoothing, quantization and noise is presented in Fig. 9, Fig. 10 and Fig. 11 respectively. Fig. 9 shows that under smoothing attack, the proposed scheme has higher robustness than [10], [13] but lags behind [17] when number of iterations are increases more than 40 . Under quantzation attack the proposed scheme has 


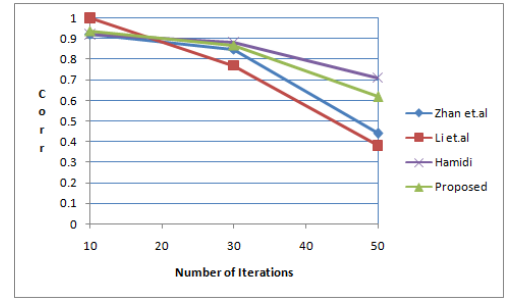

Fig. 9. Comparison of Corr Values of Proposed Scheme with [10], [13], [17] under Smoothing Attack .

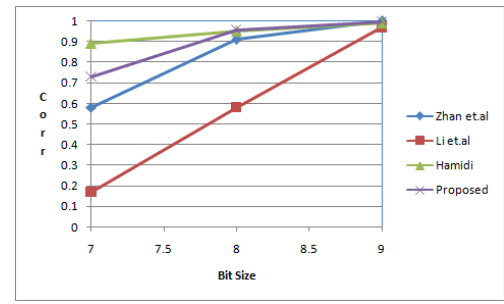

Fig. 10. Comparison of Corr Values of Proposed Scheme with [10], [13], [17] under Quantization Attak .

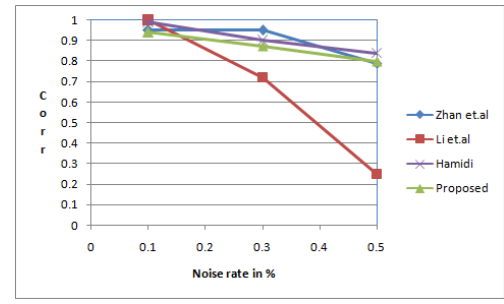

Fig. 11. Comparison of Corr Values of Proposed Scheme with [10], [13], [17] under Noise Attack.

better performance than [10], [13] and is at par with [17] as shown in Fig. 10. The proposed scheme is more robust than [13] under noise attack and passable in comparison to schemes in [10], [17]. Similarly, an overall comparison of imperceptibility is presented in Fig. 12, Fig. 13 and Fig. 14. From these figures, it can be observed that the proposed scheme has appreciably lower MRMS values than [10], [13], [17] under smoothing, quantization and noise. This indicates that the proposed scheme has higher imperceptibility. Hence, from the comparative study we can conclude that the proposed scheme shows overall improved performance than [10], [13], [17] in terms of imperceptibility, robustness and has higher security.

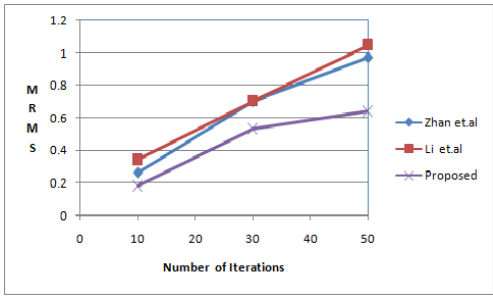

Fig. 12. Comparison of MRMS Values of Proposed Scheme with [10], [13], [17] under Smoothing Attack .

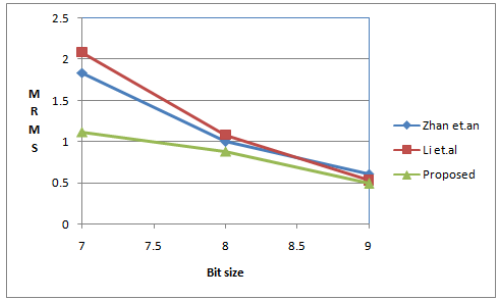

Fig. 13. Comparison of MRMS Values of Proposed Scheme with [10], [13], [17] under Quantization Attack.

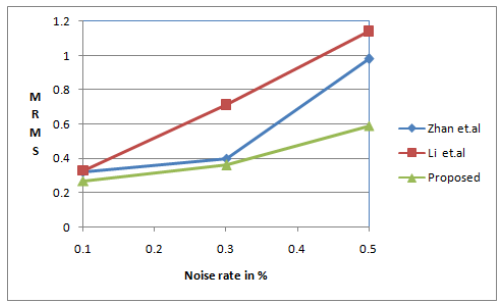

Fig. 14. Comparison of MRMS Values of Proposed Scheme with [10], [13], [17] under Noise Attack.

\section{CONCLUSION}

In this paper, a blind, robust, and secure 3-D mesh watermarking scheme is proposed that embeds watermark bits by modulating the radial distance distribution of deeper vertices using histogram mapping function. A unique watermark sequence is generated using MD5 hash function according to the grayscale watermark/logo to ensure higher security. Experimental evaluation of watermarked 3-D models reveals that no significant distortion is induced by watermarking process hence, the visual quality is good. Also, that the proposed scheme is robust against various common attacks like smoothing, additive noise, quantization, similarity transformation, cropping and simplifi- cation. Comparative analysis reflects that the proposed scheme outperforms the existing schemes and may find potential solution to the copyright pro- tection applications. Since watermark bits are embedded in deeper vertices of 3-D mesh, this scheme may not be effective for models with only positive mean curvature or flat models. This can be seen as a scope for future work. In future we wish to improve robustness of the proposed scheme using hybrid transforms.

\section{REFERENCES}

[1] Sagarika Borah and Bhogeswar Borah, " A blind, semi-fragile 3d mesh watermarking algorithm using minimum distortion angle quantization index modulation (3d-mdaqim)," in Arabian Journal for Science and Engineering, vol. 44(4), pp. 3867-3882 (2019)

[2] Hung-Kuang Chen and Wei-Sung Chen,"Gpu-accelerated blind and robust $3 \mathrm{~d}$ mesh watermarking by geometry image," Multimedia Tools and Applications., pp. 1-20 (2015)

[3] Ola M El Zein, Lamiaa M El Bakrawy, and Neveen I Ghali, "A robust 3d mesh water- marking algorithm utilizing fuzzy c-means clustering," Future Computing and Informatics Journas, vol.2(2), pp. 3867-3882 (2017)

[4] OH Khalil, Ahmed Elhadad, and A Ghareeb, "A blind proposed 3d mesh watermarking technique for copyright protectionr," The Imaging Science Journal., vol. 68(2), pp. 90—996(2020). 
[5] N Medimegh, S Belaid, and N Werghi, "A survey of the $3 d$ triangular mesh watermarking techniques," Int J Multimed.,1(1) (2015)

[6] Qilong Zhang, Xiaoying Song, Tao Wen, and Chongguo Fu, " Reversibility improved data hiding in $3 \mathrm{~d}$ mesh models using prediction-error expansion and sorting," Measurement, vol. pp.135,738-746 (2019)

[7] Neha Sharma and Jeebananda Panda, "Statistical watermarking approach for 3d mesh using local curvature estimation," IET Information Security, vol.14(6),pp. 745-753 (2020)

[8] Nassima Medimegh, Samir Belaid, Mohamed Atri, and Naoufel Werghi., "Statistical 3d watermarking algorithm using non negative matrix factorization.", Multimedia Tools and Applications,vol. 79(35), pp. 2588925904 (2020)

[9] Shinichi Nakazawa, Sho Kasahara, and Shigeo Takahashi, "A visually enhanced approach to watermarking 3d models," In Intelligent Information Hiding and Multimedia Signal Processing (IIH-MSP), pp. 110-113 (2010)

[10] Yong-zhao Zhan, Yan-ting Li, Xin-yu Wang, and Yi Qian, "A blind watermarking algo- rithm for $3 \mathrm{~d}$ mesh models based on vertex curvature," Journal of Zhejiang University SCIENCE C.,vol. 15(5), pp.351-362 (2014)

[11] Jae-Won Cho, Rmy Prost, and Ho-Youl Jung, "An oblivious watermarking for 3-d polygo- nal meshes using distribution of vertex norms," IEEE Transactions on Signal Processing, vol.55(1), pp. 142-155 (2007)

[12] Kai Wang, Guillaume Lavou'e, Florence Denis, and Atilla Baskurt, " Robust and blind mesh watermarking based on volume moments.," Computers and Graphics, vol. 35(1), pp. 1-19 (2011)

[13] Song Li, Rongrong Ni, and Yao Zhao, " A 3d mesh watermarking based on improved vertex grouping and piecewise mapping function.",emph Journal of Information Hiding and Multimedia Signal Processing.,vol. 8(1), pp. 97-108 (2017)

[14] Bata Vasic and Bane Vasic, "Simplification resilient ldpc-coded sparseqim watermarking for 3d-meshes.", emph IEEE Transactions on Multimedia., vol. 15(7), pp.1532-1542 (2013)

[15] Alaa E Abdallah, Emad E Abdallah, Mohammad Bsoul, Ahmad AlKhasawneh, and Lina Amer, "A blind 3d watermarking technique using spherical coordinates and skewness measure. ", emphInternational Journal of Security and Networks., vol. 10(1), pp. 1-8 (2015)

[16] Nassima Medimegh, Samir Belaid, Mohamed Atri, and Naoufel Werghi, " $3 \mathrm{~d}$ mesh water- marking using salient points.", emph Multimedia Tools and Applications., vol. 77(24), pp. 32287-32309 (2018)

[17] Mohamed Hamidi, Aladine Chetouani, Mohamed El Haziti, Mohammed El Hassouni, and Hocine Cherifi, " Blind robust 3d mesh watermarking based on mesh saliency and wavelet transform for copyright protection.", emph Information. , vol. 10(2) pp. 67 (2019)

[18] Xiaoqing Feng, Wenyu Zhang, and Yanan Liu, “ Double watermarks of $3 \mathrm{~d}$ mesh model based on feature segmentation and redundancy information.", Multimedia tools and ap- plications., vol. 68(3), pp. 497-515 (2014)

[19] Seung-Min Mun, Han-Ul Jang, Do-Gon Kim, Sunghee Choi, and Heung-Kyu Lee, “ A robust $3 \mathrm{~d}$ mesh watermarking scheme against cropping.”, In 3D Imaging (IC3D), pp. 1--6. (2015)

[20] Mourad R Mouhamed, Mona Mohamed Soliman, Ashraf Darwish, and Aboul Ella Has- sanien, " A robust and blind 3d mesh watermarking approach based on particle swarm optimization. ", International Journal of Sociotechnology and Knowledge Development (IJSKD), vol. 12(1), pp. 24-48 (2020)

[21] Han-Ul Jang, Hak-Yeol Choi, Jeongho Son, Dongkyu Kim, Jong-Uk Hou, Sunghee Choi, and Heung-Kyu Lee, " Cropping-resilient 3d mesh watermarking based on consistent segmentation and mesh steganalysis.", Multimedia tools and applications., vol. 77(5) pp. 5685- 5712 (2018)

[22] Hitendra Garg and Suneeta Agarwal, " A secure image based watermarking for $3 \mathrm{~d}$ polygon mesh.”, SCIENCE AND TECHNOLOGY., vol. 16(4), pp.287-303 (2013)

[23] Jianhua Wu and Leif Kobbelt, " Efficient spectral watermarking of large meshes with orthogonal basis functions.", ph The Visual Computer, vol. 21(8-10), pp. 848-857 ( 2005)

[24] Liu Jing, Wang Yinghui, He Wenjuan, and Li Ye, " A new watermarking method of 3d mesh model.", Indonesian Journal of Electrical Engineering and Computer Science., vol. 12(2), pp. 1610-1617 (2014)

[25] Nour Ashoub, Ahmed Emran, Hassan Ibrahim Saleh, et al, " Nonblind robust 3d object watermarking scheme.", Arab Journal of Nuclear Sciences and Applications., vol. 51(4), pp. :62-71 (2018)

[26] Hitendra Garg, Gagandeep Arora, and Komal Bhatia, "Watermarking for 3-d polygon mesh using mean curvature feature.”, In Computational Intelligence and Communication Networks (CICN), 2014 International Conference on, pp. 903-908. IEEE (2014)

[27] Mark Meyer, Mathieu Desbrun, Peter Schr"oder, and Alan H Barr, "Discrete differential- geometry operators for triangulated 2-manifolds.", In Visualization and mathematics III., pp. 35-57. Springer (2003)

[28] Paolo Cignoni, Massimiliano Corsini, and Guido Ranzuglia. Meshlab, “An open-source 3d mesh processing system.", Ercim news, vol. 73(4546), pp. 6 (2008)

[29] Gabriel Taubin et al., " Geometric signal processing on polygonal meshes.", Eurographics State of the Art Reports., vol. 4(3), pp. 81-96 (2000)

[30] Kai Wang, Guillaume Lavou'e, Florence Denis, Atilla Baskurt, and Xiyan He, " A bench- mark for $3 \mathrm{~d}$ mesh watermarking.", In Shape Modeling International Conference (SMI), pp. 231-235. IEEE (2010)

[31] Xifeng Gao, Caiming Zhang, Yan Huang, and Zhigang Deng, “ A robust high-capacity affine-transformation-invariant scheme for watermarking 3d geometric models. ", ACM Transactions on Multimedia Computing, Communications, and Applications (TOMM), vol. 8(2S), pp.34 (2012) 